\title{
COMMENTARY
}

\section{Complexity in Health: Can Design Help Support Interdisciplinary Solutions?}

\author{
Ledia Andrawes, ${ }^{a}$ Tracy Johnson, ${ }^{b}$ Michael Coleman ${ }^{c}$
}

\section{Key Messages}

- The collaborative nature of design practices is wellsuited to creating the conditions for productively integrating varied disciplines to solve challenging problems in global health.

- The tensions that can arise from merging different disciplines and approaches within public health and beyond, rather than hinder progress, can be surfaced for stronger solutions to emerge.

- We explore 3 productive tensions that result from integrating global health and design:

1. Integrating explicit and implicit knowledge

2. Challenging linearity with iteration

3. Enabling collective ownership of processes and solutions

- For these tensions to be productive, design and global health practitioners must do more to be open and adapt to the heritage of other disciplines; both those that have always played a role in global health and those that are just beginning to garner attention in the field.

- Designers can do more to establish early that they are not there to replace but rather to "accompany" other disciplines through collaboration.

\section{INTRODUCTION}

lobal public health programming has become in-

Greasingly complex. Overlapping investments aim at developing health policy, extending the reach of supply chains, supporting more effective service delivery, and addressing demand-related barriers to improve health. Whether it's vaccine hesitancy, antimicrobial resistance, or health care worker motivation, there is an increasing recognition that many of the problems facing the global health field have human behavioral

\footnotetext{
${ }^{a}$ Sonder Collective, London, United Kingdom.

${ }^{\mathrm{b}}$ Bill \& Melinda Gates Foundation, Seattle, WA, USA.

${ }^{\mathrm{C} C}$ Common Thread, Dublin, Ireland.

Correspondence to Michael Coleman (mike@gocommonthread.com).
}

dimensions that are often poorly understood or addressed. While this recognition has driven many global health program strategists to increasingly integrate qualitative and participatory approaches in program design as well as mixed methods for evaluation, a premium continues to be placed on global health professionals with substantive expertise-often biomedical and heavily quantitative-over the experiential and contextual insights that emerge from direct engagement with end users.

There is general agreement that the global coronavirus disease (COVID-19) pandemic has exposed and accentuated entrenched social inequities, revealing once again how vulnerable population groups-whether based on gender, disability, age, ethnicity, or geography, among others-are disproportionately affected. ${ }^{1}$ The Bill \& Melinda Gates Foundation's 2020 Goalkeepers Report summarized this concept well ${ }^{2}$ :

In the blink of an eye, a health crisis became an economic crisis, a food crisis, a housing crisis, a political crisis. Everything collided with everything else.

Health is contextual—social, cultural, and behavioraland the difficulties of taking into account more upstream systemic and social considerations have required global health programming to focus downstream, on individual behavior change and individual drivers of seeking, adopting, and adhering to treatment. ${ }^{3}$

The potential value of integrating varied disciplines to bring new insights and solutions to complex challenges is well-accepted in principle in global health. We argue in this commentary that more can be done. Drawing on the concepts and expertise of different disciplines does not automatically make a project interdisciplinary. True interdisciplinarity involves integrating information, concepts, tools, and rules that are used or produced by different disciplines on a particular subject. One might think about it less as forming a band and more as forming an orchestra where the musicians trade instruments. Yet, as we discuss in more detail, greater clarity on what the effective integration of disciplines looks like is needed. A clear and replicable process to support exactly how disciplinary boundaries can be minimized would help teams to identify unique solutions necessary for engaging the social, political, economic, and behavioral foundations that determine population health. ${ }^{4-6}$ Interdisciplinary 
The collaborative focus of design can strengthen interdisciplinary ways of working in complex settings by creating the conditions for multiple voices to be heard, considered, and effectively integrated into problem-solving approaches.

\section{Simply drawing on the concepts of different disciplines does not automatically make a project interdisciplinary.}

practice is hard work, demanding "constant explanation, adaptation and scientific readjustment" from all practitioners involved-from those engaged in project planning to research and to problem solving. ${ }^{7}$

Design practices are inherently collaborative. The process, mindset, and approach put end users and their context at the center. Design practices encourage shared understanding among diverse areas of expertise and experience. Individuals, communities, and organizations are active partners in the design and implementation of solutions. This collaborative focus of design can strengthen interdisciplinary ways of working in complex settings by creating the conditions for multiple voices to be heard, considered, and effectively integrated into problemsolving approaches. For the value of design to be optimized, designers can do more to establish early that they are not there to replace but rather to accompany other disciplines through collaboration. In this commentary, we argue that design can create a neutral space and provide a proven process for interdisciplinarity in global public health.

\section{MAKING THE CASE FOR INTERDISCIPLINARITY}

This landscape in all its beauty, sometimes gentle, sometimes terrible, cannot be seen fully by any one of the occupants of the room. Indeed, it cannot be known fully by a whole generation of men [and women]. Explorers of each generation travel into its unknown recesses and, with luck, return to share their discoveries with us. So the life of the new room would go on-thought, reflection, contemplation-as the explorers bring back their discoveries to share with the room's occupants. This landscape that we gaze on and try to understand is an epic portion of the human experience. $-\mathrm{Mead}^{8}$

Mead's plea for a "new room" in that "vast and rambling" house called science reminds us that the landscape of inquiry and problem solving cannot be seen fully by any one discipline. Recognizing that complex problems are not so neatly organized within disciplinary demarcations, the field of global health has sought to work in more interdisciplinary ways throughout its history, although not without some challenges. Embracing interdisciplinarity requires a shift among practitioners to overcome disciplinary specificities, including temporal conflicts in data collection and analysis, the requirements of institutional and disciplinary affiliations, and contrasting theoretical frameworks and methodologies. When successfully overcome, experts can bridge disciplinary divides, propel the collective effort to address the multifactorial drivers of health problems, and together identify new levers for change. Moreover, the unique knowledge that emerges from the dissolution of disciplinary boundaries is essential for addressing imperatives such as equity in human health.

Interdisciplinary training in medical schools, for example, is increasingly encouraged for specialists to consider the needs of patients more holistically. An overly specialized approach can miss valuable insight from the connections between symptoms. ${ }^{9}$ In global health, it is also common for specialists to come together to share expertise, knowledge, and skills to positively impact individual and societal health outcomes.

The case for interdisciplinary teamwork is to enable the integration of knowledge that supports a more thorough understanding of the whole picture, what Marilyn Stember calls the "holistic complex of interrelationships." ${ }^{10}$ There is no doubt that specialization along with widening the aperture of what is considered expertise has brought great strides in advancing the field of global health. Yet when navigating some complex problems, it demands not only harnessing diverse skills and knowledge but also blending disciplinary boundaries toward a common goal.

As the drive for specialists to come together to impact individual and societal health outcomes grows, so too have the range of terms used to characterize collaborative working arrangements between practitioners from different disciplines. Terms such as intradisciplinary, multidisciplinary, crossdisciplinary, interdisciplinary, and transdisciplinary are used to refer to both different types of teams and different processes within them (Figure). Yet, these terms are often unclear as to how they distinguish between different degrees of collaboration and knowledge integration.

Stember offers an overview of the different degrees of collaboration and knowledge integration within or across disciplines. ${ }^{10}$ Along the spectrum of intra- to transdisciplinary, one can see the range: from maintaining intellectual frameworks from a single discipline, to considering other disciplinary points of view, to collaborating with different disciplines, to integrating and synthesizing knowledge from other disciplines, and finally to unifying intellectual frameworks beyond disciplinary boundaries.

Given the number of terms to describe this process of working together, it is understandable that as practitioners, we are not always able to fully reflect on the processes to achieve such collaboration. However, simply drawing on the concepts of different disciplines does not automatically 
FIGURE. Degrees of Knowledge Integration Within or Across Disciplines ${ }^{a}$

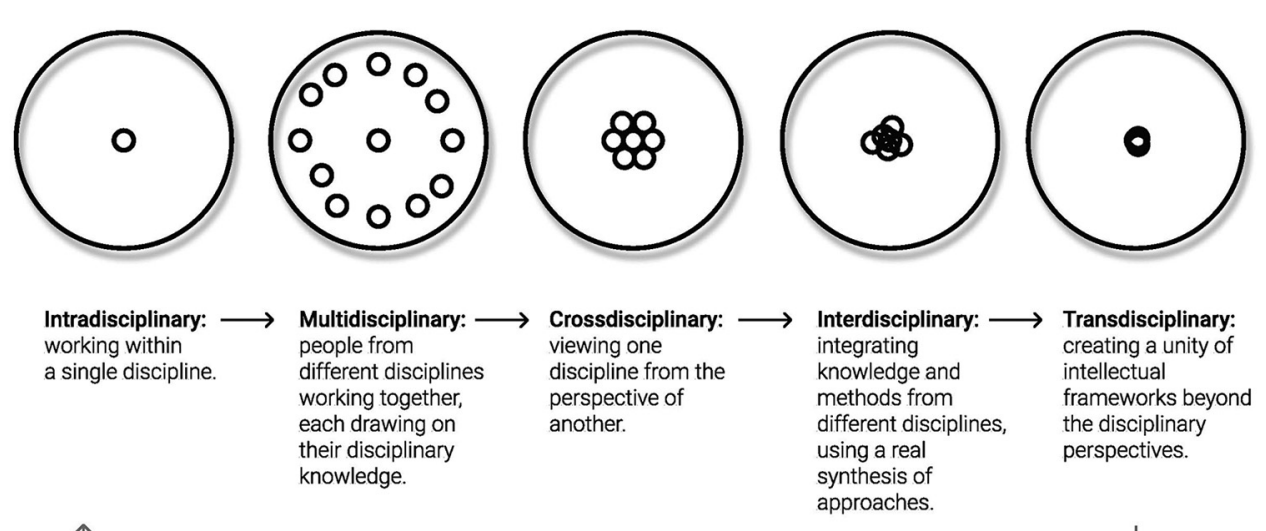

adapted from Stember. ${ }^{10}$

make a project interdisciplinary. Going beyond just assembling different types of knowledge, interdisciplinarity is a critical stance in the effort to produce a whole that is greater than the sum of the parts. With interdisciplinarity, practitioners seek out the complexity of interrelationships and integrate the contributions of other disciplines into their own. They do this in ways that dissolve traditional boundaries and open space for new insights and solutions to emerge. ${ }^{11}$

Interdisciplinarity within global health projects has been questioned before. ${ }^{5}$ On the far end of the spectrum is what some have termed "bestpracticitis"12 - a top-down, "plan and control" approach that more immediately responds to the needs of aid organizations. The argument here is that the development system relies more heavily on practitioners looking for the single right answer rather than a set of diverse solutions, spending more time trying to do things right than determining if they are doing the right things, and creating "how-to" guides and off-the-shelf toolkits that take precedence over attempts to change ways of working through deeper interaction and dialogue.

One does not need to embrace the concept of "best-practicitis" to agree that the global development system has struggled to incorporate more adaptive and integrative approaches, and in so doing has unintentionally reinforced the traditional linearity of problem definition, solution identification, implementation, and evaluation. ${ }^{12}$ Heterogeneous forms of evidence present methodological challenges that perhaps unintentionally reduce willingness to use experiential and relational methods. ${ }^{13}$
More established definitions of success have predominantly fixated on speed, scale, costs, and other critical quantifiable indicators such as intervention coverage and mortality. While these are essential features that the global health field rightly relies upon to ensure that the appropriate standards of quality, safety, and do no harm are met, this approach has conditioned many practitioners to adopt quantitative-heavy practices from their technical areas of expertise, which then perpetuate top-down cultures of procedural quantification and reporting. ${ }^{14}$ This model of impact is not well-suited to accounting for how people's experiences of the world and their health shape their knowledge and behavior. Design processes that support the co-construction of knowledge can complement traditional processes with the addition of more holistic and diverse forms of evidence. This complementarity can support practitioners to navigate global health challenges that require a deeper understanding of how to design interventions that take into greater account the complexity of human behavior.

\section{DESIGN AS A FACILITATOR OF INTERDISCIPLINARITY}

There is general agreement that interdisciplinarity practices can add value to the complex challenges facing global public health. ${ }^{4,5}$ Yet, as Kivits et al. ask, how do we do this? ${ }^{7}$

We contend that design is well-suited to creating the conditions for multiple voices to be heard, considered, and more fully integrated when problem
Design is wellsuited to creating the conditions for multiple voices to be heard, considered, and more fully integrated when problem solving in interdisciplinary teams. 
solving in interdisciplinary teams. The generative nature of design practices brings a unique form for problem solving. In the last few decades, the design field has expanded its role from shaping a product for a human to shaping relationships between humans in a system. ${ }^{15}$ This role increasingly includes interdisciplinary inquiry that tackles complex sociocultural challenges. ${ }^{14}$

Design practices can offer experiential and relational ways for looking, listening, sharing, and learning. The collaborative nature of design practices can provide strong guide rails for effectively bringing varied disciplines together to solve problems at different levels of magnification, focus, and interpretation-from the historical context to social norms and right down to the decision point. In settings where various disciplines come together for the common purpose of improving health, design processes reconceptualize social needs by illuminating the why and the how of human behavior and offering collaborative spaces for imaginative solutions. ${ }^{16}$ As many of the articles in this Supplement issue discuss, designers are increasingly expected to operate in ways that bring in more systems thinking, visualize problems and make conceptual ideas concrete, gather and mediate diverse stakeholders, and give prominence to the voice of the people behind particular global health challenges. ${ }^{14}$ By supporting action that starts from people's agency and relationships, design practices can offer a more human-centered practice for interdisciplinary working.

The need to articulate design's place among tech-

Some design
practices can treat
tensions that arise
between
disciplines as
fertile ground to
push beyond insti-
tutionalized
expectations of a
solution.
nical experts was foreseen by Richard Buchanan as he addressed the Design Educators Forum of South Africa in $2000^{17}$ :

Our bigger challenge is to explain why design is different from other "subject-based" disciplines, how it integrates knowledge from many other disciplines, and how it turns theoretical understanding in other disciplines into valuable products that can have great impact on society.

Now, 20 years later, the need for design to distinguish and establish itself alongside subjectbased disciplines that he highlighted has yet to be addressed. This articulation is needed if design is to effectively contribute to global health by creating space and a process for knowledge integration from many disciplines.

Design efforts in the international development, global health, and social innovation spaces have had a long history of embracing multiple disciplines in their practice. Bannon emphasizes that design research and practice have evolved thanks to influences from human factors and humancomputer interaction, the methodological contributions of anthropologists and sociologists, organizational information systems research, user-centered design, participatory design, and the more craft-oriented design professions. ${ }^{18}$ Design efforts focused on social innovation, ${ }^{19}$ international development, ${ }^{20-22}$ and global health ${ }^{23,24}$ continue to push the potential for interdisciplinary practice in new directions.

These trends shed some light on why a growing number of institutions have either sought out partners to contribute design expertise or have started to build internal design capacity of their own. ${ }^{25}$ Design practices are supporting the shift toward working cultures that allow for integration across the many disciplines devoted to an understanding of human behavior and human values. ${ }^{26}$ For some, integration comes with risks, such as the risk of design practices being reduced to a toolkit approach that can lead to known or obvious conclusions or overweighting individual views at the risk of alienating others. ${ }^{27-30}$ Furthermore, some design practices have been critiqued for not routinely adhering to ethical research requirements to protect research subjects and their data, which are common practices in the social sciences. ${ }^{28}$

The contrasting methodologies of different disciplines highlight tensions between their respective ways of working. However, rather than see these tensions as impediments, some design practices can treat them as fertile ground to push beyond institutionalized expectations of a solution and to open a space for new opportunities that can lead to creative solutions that are more suited to addressing the messy complexity of people's expectations, behaviors, needs, and wants related to their health. Bazzano et al. state the $\mathrm{e}^{31}$ :

central tenets of design thinking research, like iteration, tolerance for ambiguity, pivots, and rapid prototyping, are inherently at odds with some prevailing processes in health and biomedicine, particularly public health, where hypothesis-driven research is the norm.

Design practices can foster greater interdisciplinarity by harnessing such tensions. We explore 3 productive tensions that result from integrating global health and design: (1) integrating explicit and implicit knowledge, (2) challenging linearity with iteration, and (3) enabling collective ownership of processes and solutions. 


\section{- HARNESSING PRODUCTIVE TENSIONS BETWEEN DESIGN AND GLOBAL HEALTH}

\section{Productive Tension 1: Integrating Explicit and Implicit Knowledge}

In many disciplines, knowledge is often seen as taking an explicit form: things that are written down, defined, categorized, systematized, or quantified. In contrast, knowledge in design is often seen as tacit and implicit: rather than something to be articulated, knowledge exists as embodied in people's behaviors. Design practices seek to challenge the argument that knowledge only counts when it is objective, scientific, statistically valid, or considered "best-practice." It broadens what constitutes knowledge, including the experience of end users and others. Knowledge that emerges from research and observation generated through standardized (mainly quantitative) methods tends to be valued over other types of knowledge that uses qualitative, participatory, and observational techniques that by nature can be adapted as the research questions evolve. This can create tension with design practices that tend to prioritize relational and tacit types of knowledge derived more directly from the end users' points of view and which consider this type of knowledge a necessary component in addressing public health challenges.

In a project to redesign the strategy for national public health insurance in Kenya, a design-led approach was chosen by the World Bank Group and the Kenyan Government's National Hospital Insurance Fund (NHIF) to determine how NHIF could better meet the needs and preferences of the informally employed to achieve greater health insurance uptake and retention. During the 5 -month design process, more than 124 people were involved, including 84 citizens, 22 NHIF managers and staff, and 18 stakeholders from health service delivery organizations. The interdisciplinary team comprised 2 designers, 1 sociologist, 1 health economist, 1 marketing specialist, and 1 community mobilizer."

Initially, the various actors involved "labeled" the citizens involved in the project differently.

- The project's funder labeled them recipients or beneficiaries.

- The NHIF and the marketing specialist on the team considered them customers.
- The sociologist perceived them as research informants.

- The designers saw them as service-users and co-designers.

As the project progressed, the designers challenged the role of citizens as passive recipients or informants in the eyes of others by inviting citizens to join design workshops as codesigners of possible future scenarios alongside policy makers, NHIF implementers, and the interdisciplinary project team. One of the global health specialists responsible for funding the project shared how design practices ${ }^{24}$ :

...highlighted the issues from a lived experience perspective and codified the project in the language used by real people. - Participant working for a funding agency

The more participatory emphasis of the design process led the way for a diverse team of project stakeholders to ascribe greater value to the citizens' tacit knowledge and lived experience. In this case, design succeeded in helping the global health specialist reach her particular goal ${ }^{24}$ :

What I've been trying to push is how do we understand users even before we get into defining what the problem is... [this organization] is no different to other places in this regard, we've made a decision ahead of time, what are the problems. -Participant working for a funding agency

Unfortunately, there was no formal evaluation of whether the solutions proposed through this project increased access to insurance by the informal sector. However, the participants from the World Bank and NHIF senior managers credited the design-led process that guided the work to change the minds of internal NHIF stakeholders on what they needed to "solve for" as an organization and to foster more interdisciplinary collaboration among previously competitive project teams.

This expansion of what constituted knowledge went beyond traditional disciplinary boundaries such that rather than starting with a technical solution, design practices helped ensure project parameters were grounded in a deeper understanding of how people's experiences shape their consciousness and drive their health-related behaviors. However, this process can invite ambiguity into projects, and forcing people to engage with more ambiguity than they are used to creates tension. Asking experts to re-examine, reconsider,

\section{Design practices seek to challenge the argument that knowledge only counts when it is objective, scientific, statistically valid, or considered "best-practice."}

The more participatory emphasis of the design process led the way for a diverse team of project stakeholders to ascribe greater value to the citizens' tacit knowledge and lived experience. 
and blend their knowledge with that of others requires trust and a willingness to explore a problem anew. Specialists who have been working in their space for many years can feel their expertise is being shifted from the center to the periphery.

Experience-based and visual design practices can situate the knowledge of users and collaborators in dynamic ways for others to consider again. Visual design practices can bring clarity to diverse teams on otherwise complex and unfamiliar concepts. By turning complex information into sketches, models, and interactive role-plays, such design artifacts embody knowledge that is not as easily communicated using tables, words, and numbers. Many global health practitioners often stop at this point, seeing the role of design to fulfill that communication function. But design's value comes in its ability to challenge assumptions based on technical expertise alone and instead to create opportunities for explicit knowledge to blend with user perspectives and other types of tacit knowledge. Design practices can further support practitioners in acknowledging where their knowledge sits in relation to others and facilitating teams to collectively move beyond any unrecognized biases. ${ }^{32,33}$ Resituating a type of knowledge in relation to others enables practitioners to build on the tradition of participatory methods in global health and relate to beneficiaries less as subjects of inquiry and more as collaborators in the desired change.

\section{Productive Tension 2: Challenging Linearity With Iteration}

Many approaches suggested for effectively navigating complex problems contradict what can be a fairly rigid and linear process of problem definition, solution identification, implementation, and evaluation. ${ }^{12,34}$ While such linear processes can be efficient, they have proven less effective in complex situations where problem definition remains an ongoing, open, and critical reflection throughout the project, rather than just an upfront phase. In contrast to a linear-thinking, single-solution approach born of analysis, an iterative design approach is flexible in nature, continuously skeptical as to the definition of the problem itself, opportunistic in its generation of solutions, and almost obsessed with introducing creative options and experimentation. Such an approach challenges the notion that there is 1 pathway to change. challenges the notion that there is one pathway to change. solving involves regular cycles of learning and experimentation to reach a solution. ${ }^{35}$ Given the pace of funding cycles that drive for more rapid solution development, a tension arises with design practices that can insist on longer processes of iteratively working through the complexity of the problems. $^{\dagger}$

For people who seek the certainty of externally structured, well-defined problems, iterative design processes have the potential to create discomfort for people who are not used to them. Take, for example, a 5-day design sprint/workshop in Zimbabwe that aimed to use mixedmethods, segmentation-based insights to generate innovative ideas and early prototypes to address poor uptake of voluntary medical male circumcision (VMMC) services. There were approximately 40 people in the sprint/workshop, including policy representatives from the Ministry of Health, practitioners from partner organizations with various technical and programmatic backgrounds, and current and potential clients of VMMC services.

One of the project sponsors reflected afterward on how challenging it was for her to let go of control. This was particularly the case with unproven activities the design team used to push participants into a more creative space when generating ideas. For example, an activity provided participants with several rounds of unrealistic scenario prompts that started with "what if. .." or "imagine if. .." for them to generate more novel ideas. The different ideas were rotated around the room, and participants were invited to build upon those that were generated by others. These less "evidence-based" methods and more creative scenario-based methods to generate additional ideas were difficult for this project implementer to accept on day 2. By day 5, the implementer declared the week as a significant success as it pushed teams to think creatively, work in more interdisciplinary ways, and develop new prototypes that could be further tested and developed.

At the same time, there were moments during the week that the project implementer felt nervous and questioned whether bringing in a design team was a mistake. This implementer shared with an author ${ }^{24}$ :

The approach is the approach, I still can't cope with the chaos part of it, but the approach is the approach. -Implementer involved in the project

${ }^{\dagger}$ Plan-Do-Study-Act and adaptive management approaches are attempting to bring similar iterative working styles into global health practice. These efforts are emergent and perhaps would be a fruifful avenue for collaboration with design. 
This discomfort usually occurs when diverse teams are forced to engage with the ambiguity, disorder, and messiness of integrating each other's knowledge and ideas. Design practices can help global health practitioners navigate ambiguity and open deliberative and adaptive spaces for new possibilities to emerge. ${ }^{36}$ This occurs through experiential and speculative processes, such as ideation and prototyping, that support interdisciplinary teams in maintaining the "parallel lines of thought" necessary for integrating analytical and creative perspectives 37 and the "double vision" that helps a designer be both learner and creator. ${ }^{38}$

This movement between the different activities associated with learning, analyzing, and doing are critical characteristics of design when seeking interdisciplinary solutions because answering the wrong question or answering the right question poorly is increasingly costly in complex settings. ${ }^{39}$ However, designers need to negotiate a balance between practices that are human-centered and messy and practices that are predictable, bounded, and meet the institutional and project needs. Despite the aspirations and expectations of some, design practices do not always offer implementers immediate answers to complex problems. Sometimes, as they did in this situation, they provide new and welcome ways of collaboratively navigating intractable problems that persist with no obvious solutions.

\section{Productive Tension 3: Enabling Collective Ownership of Processes and Solutions}

Many global health practitioners are inspired by the sense of possibility that emerges when a community adopts a solution as their own. The global Ebola virus disease and COVID-19 pandemics have demonstrated that to achieve impact and scale solutions that are sustainable over time, global health practitioners must design solutions with local communities and not simply for them. What constitutes collective ownership can be difficult to clearly define within projects that involve diverse stakeholders with varied agendas. At the same time, there is a growing consensus that enabling collective ownership ${ }^{40}$ :

...demands genuine interactions, creating enabling conditions and spaces for incremental changes, and building shared values.

The collaborative nature of design practices supports interdisciplinary work by creating spaces that call for the exchange of values-based ideas and nurture a greater sense of collective ownership. Designers can provide a structure for continuous interdisciplinary collaboration by (1) bringing in dialogue-based design methods, (2) stimulating the creativity and ideas of collaborators, and (3) enabling collaborators to bring in their material and intellectual culture. ${ }^{41}$ This suggests that practicing design in interdisciplinary settings can require additional skillsets as designers are also required to be fluent in balancing multiple participant ideas ${ }^{42,43}$; addressing "changing roles of power" in groups ${ }^{44}$; and developing contextually adapted methods for diverse participants to contribute throughout a process. ${ }^{45}$ Design practices that seek to integrate multiple (and sometimes contradictory) viewpoints that are centered around the end user experience can come into tension with a more multidisciplinary approach in global health-an approach that often includes more perspectives but can unknowingly maintain disciplinary boundaries and undervalue user experience.

In the previously mentioned project seeking to redesign a citizen-centered public health insurance service in Kenya, senior NHIF managers, who would eventually implement the service, determined that collective ownership was critical to the project's success. Through a series of ethnographic activities and design workshops, stakeholders across different organizational departments, external providers, and citizens from different regions came together to provide unique insights into current challenges and future possibilities associated with such a service. Over time, a culture of reciprocity and knowledge exchange developed, which ultimately created a sense of co-ownership in the final service. One of the key project sponsors reflected on their experience with the design process ${ }^{24}$ :

I found that in this way you are able to involve all the stakeholders, and you look at the situation from all the angles... for me, that was the key thing. -Implementer perspective

The strategy that was developed was holistic in that it did not only provide the required changes in communications and messaging toward the informally employed but also covered a more holistic set of required changes. These ranged from institutional reforms for improved service quality by health care providers and the need for new financial models to support more vulnerable groups. Although it was design practices that helped to build bridges and collective ownership across different stakeholder groups, it was the commitment of individual stakeholders that was foundational to the implementation of the strategy's recommendations.

\section{Design practices can help global health practitioners navigate ambiguity and open deliberative and adaptive spaces for new possibilities to emerge.}




\section{CONCLUSION}

This commentary is an invitation to both designers and public health professionals to join forces more openly and more often to bring together the plurality of expertise within public health and the practical, people-centered, problem-solving approaches of design. For design to genuinely harness interdisciplinary solutions, it requires that practitioners of both design and global health reflect on their respective contributions to the bounded nature of global health programs.

Design will not solve all the problems we are grappling with as a global health community. Where design can contribute is with its convening power and ability to productively bring interdisciplinary teams together toward a common goal. As the articles in this Supplement issue demonstrate, when done well, design can create space for the blending of ideas untethered to narrow communities of practice, academic process, pressures, and tradition. This is an exciting opportunity to expand our definition of knowledge, embrace iteration, and foster collective ownership. Design practices can provide a people-centered framework to make the most of the diverse disciplines and expertise within public health so that they are better able to flourish collectively, creatively, and productively, such that true interdisciplinarity can be harnessed to tackle the toughest global health challenges we face.

Acknowledgments: This article draws some of its foundational positions and further adapts some of the themes discussed in Andrawes' doctoral thesis, which explored the value of design, as well as the ethical challenges and enabling conditions for applying design in global health and development. The authors would also like to express our sincere thanks for the contribution of Gael Welstead, Human-Centered Design Lead at Common Thread, who contributed her time and expertise to the research and writing of early drafts of this Commentary. We would also like to thank 2 of the guest editors, Shilpa Das and Nikki Tyler, for their helpful guidance, encouragement, and invitation to participate in this issue. Special thanks also to Anne LaFond for introducing Ledia and Tracy many years ago and being an early catalyst for the Design for Health community. The authors are grateful for the efforts of the GHSP team, including Editor Stephen Hodgins for providing invaluable perspectives on the topic. Sincere thanks to the anonymous reviewers for their constructive feedback. All this input helped make this a better article. Finally, thank you to our colleagues, family, and friends for the critiques, patience, and interdisciplinary tensions that helped us get this to the finish line.

Author contributions: Ledia Andrawes prepared the outline and coordinated co-authors and researchers Michael Coleman and Tracy Johnson. Co-authors supported with research, review, and drafting.

Competing interests: None declared.

\section{REFERENCES}

1. McNeely CL, Schintler LA, Stabile B. Social determinants and COVID-19 disparities: differential pandemic effects and dynamics. World Med Health Policy. 2020;12(3):206-217. CrossRef
2. Gates B, Gates MF. COVID-19: a global perspective. Bill \& Melinda Gates Foundation. Accessed September 14, 2021. https://www. gatesfoundation.org/goalkeepers/report/2020-report/

3. Blas E, Kurup AS, eds. Equity, Social Determinants and Public Health Programmes. World Health Organization; 2010. Accessed September 17, 2021. https://apps.who.int/iris/bitstream/handle/ 10665/44289/9789241563970_eng.pdf? sequence=1\& isAllowed=y

4. Gavens L, Holmes J, Bühringer G, et al. Interdisciplinary working in public health research: a proposed good practice checklist. J Public Health (Oxf). 2018;40(1):175-182. CrossRef. Medline

5. Van Teijlingen ER, Regmi PR, Adhikary P, Aryal N, Simkhada P. Interdisciplinary research in public health: not quite straightforward. Health Prospect. 2019;18(1):4-7. CrossRef

6. Galea S, Annas GJ. Aspirations and strategies for public health. JAMA. 2016;315(7):655-656. CrossRef. Medline

7. Kivits J, Ricci L, Minary L. Interdisciplinary research in public health: the "why" and the "how." J Epidemiol Community Health. 2019;73 (12):1061-1062. CrossRef. Medline

8. Mead M. Blackberry Winter. William Morrow; 1972.

9. Rimmer A. The UK needs more generalists, but where will they come from? BMJ. 2017;356:j1116. CrossRef

10. Stember M. Advancing the social sciences through the interdisciplinary enterprise. Soc Sci J. 1991;28(1):1-14. CrossRef

11. Klein JT. A conceptual vocabulary of interdisciplinary science. In: Stehr N, Weingart $\mathrm{P}$, eds. Practising Interdisciplinarity. University of Toronto Press; 2000:3-24.

12. Ramalingam B. Aid on the Edge of Chaos: Rethinking Internationa Cooperation in a Complex World. Oxford University Press; 2015.

13. Lennie J, Tacchi J, Wilmore M. Critical reflections on the use of participatory methodologies to build evaluation capacities in international development organisations. Paper presented at: 8th World Congress 2010 Participatory Action Research and Action Learning; September 6-9, 2010; Melbourne, Australia.

14. Inns T, ed. Designing for the 21 st Century. Volume II: Interdisciplinary Methods and Findings. Gower Publishing; 2010

15. Colussi M. Designers through borders. Design of a Collaborative System-Innovation Platform for the Sustainable Development of Emerging and Low-Income Contexts. Dissertation. Politecnico; 2010.

16. Murray R, Caulier-Grice J, Mulgan G. The Open Book of Social Innovation: Ways to Design, Develop and Grow Social Innovation. NESTA; 2010

17. Buchanan R. Human dignity and human rights: thoughts on the principles of human-centered design. Design Issues. 2001;17(3):35-39. CrossRef

18. Bannon L. Reimagining $\mathrm{HCl}$ : toward a more human-centered perspective. Interactions. $2011 ; 18(4): 50$. CrossRef

19. Brown T, Wyatt J. Design thinking for social innovation. Stanford Social Innovation Review. 2010;12(1):29-43. Accessed September 17, 2021. https://ssir.org/articles/entry/design_thinking_for_ social_innovation

20. Braund $P$, Schwittay $A$. The missing piece: human-driven design and research in ICT and development. In: International Conference on Information and Communication Technologies and Development. IEEE; 2006:2-10. CrossRef

21. Dearden A, Rizvi H. Participatory design and participatory development: a comparative review. In: PDC'08: Experiences and Challenges, Participatory Design Conference; October 1-4, 2008; Indiana University, Bloomington, IN. Accessed September 17, 2021. https://shura.shu.ac.uk/29/1/fulltext.pdf

22. Oosterlaken I. Design for development: a capability approach. Design Issues. 2009;25(4):91-102. Accessed September 17, 2021 CrossRef 
23. Holeman I, Cookson TP, Pagliari C. Digital technology for health sector governance in low and middle income countries: a scoping review. J Glob Health. 2016;6(2):020408. CrossRef. Medline

24. Andrawes, L. Encountering Design for Development: An Exploration of Design Value and Ethics in Practice. Dissertation. Institute for Global Prosperity, University College London; 2021. Accessed September 17, 2021. https://discovery.ucl.ac.uk/id/eprint/ 10132031

25. Mishra P, Sandhu JS. Design is an essential medicine. Glob Health Sci Pract. 2021;9(Suppl 2). CrossRef

26. Holeman I, Kane D. Human-centered design for global health equity. Inf Technol Dev. 2019;26(3):477-505. CrossRef

27. Norman DA. Human-centered design considered harmful. Interactions. 2005;12(4):14-19. CrossRef

28. Tolley EB. Traditional Socio-Behavioral Research and HumanCentered Design: Similarities, Unique Contributions and Synergies. FHI 360; 2017. Accessed September 17, 2021. https://theimpt. org/wp/wp-content/uploads/2017/12/documents_reports_ Report-HCD-BSS-Research.pdf

29. Cotsaftis $\mathrm{O}$. Human-centered design is bullshit. Medium. May 26 2019. Accessed September 17, 2021. https://medium.com/this-ishcd/human-centered-design-is-bullshit-2ff83d31b5cb

30. Bazzano AN, Yan SD, Martin J, et al. Improving the reporting of health research involving design: a proposed guideline. BMJ Glob Health. 2020;5(2):e002248. CrossRef. Medline

31. Bazzano AN, Martin J, Hicks E, Faughnan M, Murphy L. Humancentred design in global health: a scoping review of applications and contexts. PloS One. 2017;12(11):e0186744. CrossRef. Medline

32. Biörgvinsson E, Ehn P, Hillgren P-A. Agonistic participatory design: working with marginalised social movements. CoDesign. 2012:8(2-3):127-144. CrossRef

33. DiSalvo C. Adversarial Design. MIT Press; 2015.
34. Mosse D. Process-oriented approaches to development practice and social research. In: Development as Process: Concepts and Methods for Working with Complexity. Routledge; 1998:3-30.

35. Tushman ML, O'Reilly CA III. Ambidextrous organizations: managing evolutionary and revolutionary change. CalifManage Rev. 1996;38(4):8-29. CrossRef

36. Cipolla C, Manzini E. Relational services. Knowl Technol Policy. 2009;22(1):45-50. CrossRef

37. Lawson B. Parallel lines of thought. 1993. Accessed September 17 2021. https://www.researchgate.net/profile/Bryan-Lawson-2/ publication/257353502 Parallel lines of thought/links/ 00b7d52502662beb83000000/Parallel-lines-of-thought.pdf

38. Schön DA. The Reflective Practitioner: How Professionals Think in Action. Basic Books; 1983

39. Liedtka J, Parmar B. Moving design from metaphor to management practice. J Organ Des. 2012;1(3). CrossRef

40. Sarriot $E$, Shaar AN. Community ownership in primary health caremanaging the intangible. Glob Health Sci Pract. 2020;8(3):327331. CrossRef. Medline

41. Reitsma L, Light A, Zaman T, Rodgers P. A respectful design framework. Incorporating indigenous knowledge in the design process. Design J. 2019;22(sup 1):1555-1570. CrossRef

42. Visser W. Design: one, but in different forms. Design Stud. 2009;30 (3):187-223. Accessed September 17, 2021. CrossRef

43. Margolin V. Design for development: towards a history. Design Stud. 2007;28(2):111-115. CrossRef

44. Sanders EBN. Design research in 2006. Design Research Quarterly. 2006; 1 (1). Accessed September 17, 2021. https://imgl .wsimg. com/blobby/go/5f4a9a22-5569-4876-880a-58927836546a/ downloads/DesignResearchin2006_Sanders_06.pdf?ver= 1600007016962

45. Binder T. Setting the stage for improvised video scenarios. $\mathrm{CHI}$ ' 99 Extended Abstracts on Human Factors in Computer Systems - CHI '99. 1999:230-231. CrossRef

\section{Peer Reviewed}

Received: April 7, 2021; Accepted: August 17, 2021.

Cite this article as: Andrawes L, Johnson T, Coleman M. Complexity in health: can design help support interdisciplinary solutions? Glob Health Sci Pract. 2021;9(Suppl. 2):S217-S225. https://doi.org/10.9745/GHSP-D-21-00222

(c) Andrawes et al. This is an open-access article distributed under the terms of the Creative Commons Attribution 4.0 International License (CC BY 4.0) which permits unrestricted use, distribution, and reproduction in any medium, provided the original author and source are properly cited. To view a copy of the license, visit https://creativecommons.org/licenses/by/4.0/. When linking to this article, please use the following permanent link: https:// doi.org/10.9745/GHSP-D-21-00222 